\title{
Balancing Act: The Need for Work Style Reform for Young and/ or Female Gastroenterologists
}

\author{
Hidekazu Suzuki ${ }^{1}$ \\ Published online: 9 October 2019 \\ ○) Springer Science+Business Media, LLC, part of Springer Nature 2019
}

The notion that a physician benefits others rather than themselves has long been respected, particularly in the East. For this reason, physicians have been thought to be devoted to patients, giving priority to them over everything else. Therefore, work style reform and the work-life balance of doctors, especially among clinicians, young doctors, and female doctors, are considered the last untouched islands for work style reform among various occupations.

In this issue of Digestive Diseases and Sciences, Jang et al. [1] investigated work-life patterns via prospective data collection of the time consumed at work and home as well as occupation-related symptoms among 222 gastroenterologists. As a result, they discovered a significant correlation between work-life balance and musculoskeletal pain, gastrointestinal symptoms including Rome IV-based functional dyspepsia [2], mental illness symptom, and "burnout". They showed that among Korean gastroenterologists working in tertiary care hospitals, the mean time spent at work and at home was $71.5 \pm 19.0$ and $16.6 \pm 15.9 \mathrm{~h}$ per week, respectively. Korean doctors with and without children had working times up to $69.6 \pm 18.5 \mathrm{~h}$ per week [1], indicating doctors are working beyond the $52 \mathrm{~h}$ weekly maximum employed workers in the Korean Labor Standard Acts. Moreover, the time spent at home was significantly increased in doctors with children and even more among female doctors. In particular, female gastroenterologists spent more time with their families, despite having a workload similar to that of male doctors [1]. They pointed that this could be a dangerous signal to the Korean Medical Society, in which women accounted for more than $25 \%$ of doctors in 2017 [1]. Furthermore, they reported that $89.6 \%$ of participants suffered from musculoskeletal pain; this was experienced

Hidekazu Suzuki

hsuzuki@tokai.ac.jp

1 Department of Gastroenterology and Hepatology, Tokai University School of Medicine, 143 Shimokasuya, Isehara, Kanagawa 259-1193, Japan significantly more frequently in women. The number of colonoscopies and work/life ratio were independently related to this musculoskeletal pain severity. Furthermore, according to multivariable analysis, severe mental illness symptoms were independently affected by severe musculoskeletal pain, a high number of endoscopic procedures, and a high work/life ratio. Interestingly, the job satisfaction score was lowest among women in their forties. Fewer female doctors answered that they would reselect medicine or gastroenterology as their profession if they had a chance to select their career again compared to men [1], a shocking result that suggests the profession of gastroenterologist is undesirable among certain segments of the population. As described by the authors, since doctors that could handle their work-life balance were excluded, the gastroenterologists surveyed in tertiary care hospitals in the present study might not represent the entire population of Korean gastroenterologists. Nevertheless, it was true that the studied gastroenterologists were highly "burned out' due to long hours and laborintensive work. The burnout rate of their study population (66.7\%) was higher than that of gastroenterologists in the USA [3]. Furthermore, work-life imbalance and burnout were most severe in female doctors in their thirties and forties. Work-life imbalance in Korean gastroenterologists, particularly in young or female doctors, has recently been an important social issue discussed at major gastroenterology conferences, including Asian Pacific Digestive Week 2018 (APDW2018) and will be discussed at Korea Digestive Disease Week 2019 (KDDW2019).

As for the neighboring country, Japan, the Japanese Society of Gastroenterology (JSGE) has established a female gastroenterologists' association and has recently developed a career support committee in the name of supporting not only female doctors, but also young doctors, including interns and residents. The career support committee has been planning hands-on training to support female and young doctors in academic societies, increasing the number of female councilors, and developing childcare facilities at national 
and domestic academic meetings. Recently, the career support committee held a nationwide case study competition symposium at the annual meeting of JSGE in order to support young doctors regardless of gender and recruit young gastroenterologists to the society; it was received with great popularity by many of the participants. This activated discussions among young doctors, and the younger generation was successfully brought back to the JSGE meeting. The 2nd Career Support Committee/Branch Women's Doctor Meeting in 2019 will be held on Saturday, November 23, 2019, during JDDW2019 in Kobe, Japan. The agenda at this time is a report on the activities of women's gastroenterology associations in each regional branch of JSGE and discussions regarding the career support committee's plans for the 106th general meeting to be held in April 2020. Yet, the committee was not directly involved in issues such as the current working conditions of doctors, working hours, overtime, and childcare leave. Nonetheless, the Ministry of Health, Labor, and Welfare in Japan has currently started the process of reforming doctors' working environment, slated to be completed in 2024. They plan to implement continuous working hour regulations and inter-work interval regulations from the viewpoint of ensuring the health and medical safety of hospital doctors. Besides, they are investigating the use of objective indicators to identify doctors who are unable to secure sufficient sleep time in order to ensure their continued health. The Japanese government has begun to develop regulations for the working environments of doctors. As noted in the present paper [1], even in the world of gastroenterologists, the prevention of physician work-life imbalance and burnout is an urgent issue to address not only for the health of doctors, but also for patient safety in all nations.

Acknowledgments This work was supported by Tokai University General Research Organization Grant.

\section{References}

1. Jang ES, Park SM, Park YS, Lee JC, Kim N. Work-life conflict and its health effects on Korean gastroenterologists according to age and sex. Dig Dis Sci. (Epub ahead of print). https://doi. org/10.1007/s10620-019-05842-w.

2. Stanghellini V, Chan FK, Hasler WL, et al. Gastroduodenal disorders. Gastroenterology. 2016;150:1380-1392.

3. Lacy BE, Chan JL. Physician burnout: The hidden health care crisis. Clin Gastroenterol Hepatol. 2018;16:311-317.

Publisher's Note Springer Nature remains neutral with regard to jurisdictional claims in published maps and institutional affiliations. 